(C) 2020 IEEE

\title{
Smooth Power Direction Transition of a Bidirectional LLC Resonant Converter for DC Transformer Applications
}

J. Kucka and D. Dujic

This material is posted here with permission of the IEEE. Such permission of the IEEE does not in any way imply IEEE endorsement of any of EPFL's products or services. Internal or personal use of this material is permitted. However, permission to reprint / republish this material for advertising or promotional purposes or for creating new collective works for resale or redistribution must be obtained from the IEEE by writing to pubs-permissions@ieee. org. By choosing to view this document, you agree to all provisions of the copyright laws protecting it. 


\title{
Smooth Power Direction Transition of a Bidirectional LLC Resonant Converter for DC Transformer Applications
}

\author{
Jakub Kucka, Member, IEEE, and Drazen Dujic, Senior Member, IEEE
}

\begin{abstract}
The bidirectional LLC resonant converter operated at fixed switching frequency is a preferable candidate for a true de transformer that does not actively control the power flow. This is due to its naturally stiff voltage ratio regardless of the imposed load, its low switching losses, and a stable open-loop behavior. However, in the open-loop operation, determining the required operation direction of the converter is a challenge which needs to be addressed. This paper investigates good design practices for a smooth power direction transition and discusses different strategies for estimating the required operation direction. Moreover, it proposes a new Active Bridge Switchover (ABS) method that is based on an observation of the peak currents in the resonant tank. The proposed method is robust and does not require high precision of the current sensors. The properties of the method are studied using simulations and its practical feasibility is validated experimentally on a low-voltage prototype. This paper is accompanied by a video demonstrating the effectiveness of the ABS method in the real time operation.
\end{abstract}

Index Terms-dc transformer, solid-state transformer, DCX, LLC converter, resonant, bidirectional operation, fixed frequency.

\section{INTRODUCTION}

W ITH ever dropping prices for power electronic converters, dc grids are becoming a conceivable solution for small-to-large scale power distribution. While the development is relatively slow in utility grids [1], there are many other applications that are proactively investigating and testing dc power distribution networks. Marine applications are leading the way [2], [3] supported by several demonstration projects around the world [4], [5]. The evolution to large scale dc grids is currently prohibited by the insufficiently developed technology and the lack of standards [2]. One of the key components that is not entirely solved is the dc transformer, also referred to as dc-dc solid-state transformer, required for providing isolated interconnection between the grid areas.

In the system concept that we follow, in literature often branded as DCX, the dc transformer is a component that shares a lot of properties with the conventional ac transformer. Hence, it should behave as a passive component that naturally transfers the power according to the state of the connected grids. The required properties can be summarized as follows:

- high efficiency,

J. Kucka and D. Dujic are with Power Electronics Laboratory, EPFL, Lausanne, Switzerland, e-mail: jakub.kucka@epfl.ch,drazen.dujic@epfl.ch.

The results presented in this paper are a part of the EMPOWER project that has received funding from the European Research Council (ERC) under the European Union's Horizon 2020 research and innovation programme (Grant agreement No. 818706).
- bidirectional operation,

- galvanically isolated power transfer through a mediumfrequency transformer (MFT),

- arbitrary value of voltage ratio,

- weak dependence of the voltage ratio on the transferred power (stiff voltage-ratio loading characteristic; ideally, the voltage ratio should emulate resistive voltage drop according to converter losses to ensure most efficient power flow), and

- operation without any knowledge or data regarding the connected dc grids and without the necessity of commands from a grid operator.

Furthermore, the dc transformer should generally offer some kind of protection services such as limiting the overcurrents or an option to disconnect the grids as a solid state switch.

In literature, many isolating dc-dc converters based on dualactive bridges (DABs) [6], resonant-tank DABs [7], controlled resonant converters [8], [9] or modular multilevel converters (MMCs) [10], [11] have been proposed for such applications. Nevertheless, these solutions have to necessarily employ closed-loop control to keep the weakly damped currents of the MFT stable. Although the closed-loop control is a common well-established solution in power electronics, it implies that a power setpoint is required. This, however, means that either the grid operator must provide the power commands or that the power transfer is controlled according to some droop based on the ratio of the dc transformer voltages. Due to the relatively low precision of the available dc-voltage measurement sensors, the droops have to be set for relatively large voltage drop (several percent instead of the typical one to two percent representing the losses). As a consequence, the power flow in large scale grids with high number of parallel paths and dc transformers would not necessarily follow the path of lowest resistance. In summary, these solutions do not comply with the requirements listed above.

The second approach to the dc transformer realization is based on resonant converters operated in open loop at a fixed switching frequency near the resonant frequency. The solutions from literature [12]-[23] mostly employ some variant of the LLC resonant converter to provide basically all of the aforementioned properties. The resonant nature of the converters enables zero-voltage and zero-current switching (ZVS and ZCS) that leads to low switching losses and the operation near resonant frequency naturally achieves very stiff current-voltage characteristics without any control loops or additional grid knowledge. In the case of overload, an additional closed-loop 
controller can be theoretically implemented to limit the overcurrents through changing the duty cycles and the switching frequency (even without the loss of ZVS and ZCS [24]). The main remaining challenge is a smooth bidirectional operation, since the LLC converters are conventionally designed for unidirectional power flow. Unlike in the closed-loop operation of the LLC converters where the power direction is given by the power setpoint, one of the particular problems arising with the open-loop LLC converter operation at fixed switching frequency is the necessity to recognize in which direction the converter should be operating in that particular moment.

In literature two groups of solutions have been proposed regarding this issue:

1) observing the voltage gain of the converter to decide the power direction [9], [25] or

2) a concurrent switching at both converter sides to achieve a true seamless bidirectional operation [17]-[23].

Nonetheless, the first solution would require high-resolution offset-free measurements of the dc-link voltages to estimate the exact instant when the voltage gain reached the value indicating zero power, required for smooth direction transition. Such voltage measurement is challenging, since the voltagegain loading characteristic is very stiff. Hence, the practical solutions use some hysteresis that changes the direction of converter operation only when the voltage gain ratio reaches some value outside of the usual operating range [9]. Since such high voltage-gain mismatch would cause very high inrush currents, the direction is reversed using some method for current limiting (a soft-start scheme such as that in [26] or a closedloop current controller [9]). However, this means that the converter operated with this power reversal strategy possesses strongly non-linear and partly discontinuous behavior at low powers, which is demonstrated in [9]. This is likely to cause some stability issues at large-scale dc grids and it is even a challenge to do some large-scale study regarding this problem because of the described heavily non-linear behavior.

Despite its clear advantages regarding the quality of the power direction transition, the second solution relies on a relatively precise knowledge of the converter circuit. Since both converter sides are operated concurrently, the converter includes a parasitic resonant DAB. Hence, the tank needs to be excited at the exact resonant frequency to avoid an undesired power transfer. The stability of operation can be reinforced by a careful design of delay times between the switching signals of the bridges [21] or by an online estimation of the resonant frequency [20]. Nonetheless, the operation requires switching at both sides at all times. Even though, this can be seen as an advantage when unipolar switches (e.g. MOSFETs) are applied, since the active rectifying is likely to lower the conduction losses, with bipolar devices (e.g. IGBTs) this only increases the switching losses and thus diminishes the dc transformer efficiency. Hence, the concurrent switching is mainly advantageous for converters with unipolar devices and well-known parameters of the converter.

In this paper, a new Active Bridge Switchover (ABS) method, based on observing the peak currents at the resonant tank, is proposed. This requires only two simple ac-current sensors with low precision and leads to a robust behavior, insensitive to variation of resonant tank parameters. Furthermore, the paper proposes good practices for the design of the resonant tank for obtaining a smooth linear voltage gain loading characteristic and shows the implementation of the novel method. The feasibility of the method is demonstrated through simulations for medium-voltage high-power dc transformer and experiments on low-voltage low-power dc transformer prototype.

In the following section, Section II, the studied topology is presented and the model for the design investigations is derived. Section III investigates good practices for the LLCconverter-based dc transformer, thereby answering two questions:

- What is the most desirable switching to resonant frequency ratio for smooth converter operation?

- What is a good design practice for the design of the resonant-tank series impedance $Z_{0}$ (represented by the quality factor at the rated power $\left.Q_{\mathrm{N}}\right)$ ?

After the design practices have been set, the proposed ABS method for the decision of the operation direction is presented and explained in Section IV. The feasibility of the method (together with the design practices) is validated by simulations in Section $\mathrm{V}$ and the experimental validation on low-voltage prototype follows in Section VI. Conclusions are drawn in Section VII.

\section{Studied Topology, Model, AND Assumptions}

The dc transformer that is the subject of the studies presented in this paper is depicted in Fig. 1. The dc transformer is connected to two dc grids: Grid 1 and Grid 2 at the respective dc-link terminals. The voltages of these grids are controlled by other grid-building converters located somewhere in the grid. Since the power transfer should be truly bidirectional, without a preferred direction, the resonant tank is constructed as a symmetric CLLC with split capacitors around the MFT. This solution has been proposed for bidirectional power transfer first in [8]. The power electronics part consists of two bridges Bridge 1 and Bridge 2 that are constructed as full bridge converters. In the conventional operation presented here, only one of the bridges is actively switched at a time while the other is operating as a passive rectifier. The active bridge is operated in a complementary manner with a $50 \%$ duty cycle. Although the studies provided in this paper utilize full-bridge converters, the results apply to half-bridge converters as well.

For the design studies in this paper, the dc transformer is represented by an equivalent model depicted in Fig. 2. As can be seen, the currents and voltages of the model are related to the primary side:

$$
\begin{aligned}
& {\left[\begin{array}{c}
i_{\mathrm{r}, 2}^{\prime} \\
i_{\mathrm{dc}, 2}^{\prime}
\end{array}\right]=\frac{1}{n} \cdot\left[\begin{array}{c}
i_{\mathrm{r}, 2} \\
i_{\mathrm{dc}, 2}
\end{array}\right],} \\
& {\left[\begin{array}{c}
v_{\mathrm{r}, 2}^{\prime} \\
v_{\mathrm{dc}, 2}^{\prime}
\end{array}\right]=n \cdot\left[\begin{array}{c}
v_{\mathrm{r}, 2} \\
v_{\mathrm{dc}, 2}
\end{array}\right] .}
\end{aligned}
$$

Furthermore, the resonant capacitor $C_{\mathrm{r}}$ is assumed to be split symmetrically between the converter sides:

$$
\begin{aligned}
& C_{\mathrm{r} 1}=2 \cdot C_{\mathrm{r}} \\
& C_{\mathrm{r} 2}=n^{2} \cdot 2 \cdot C_{\mathrm{r}} .
\end{aligned}
$$




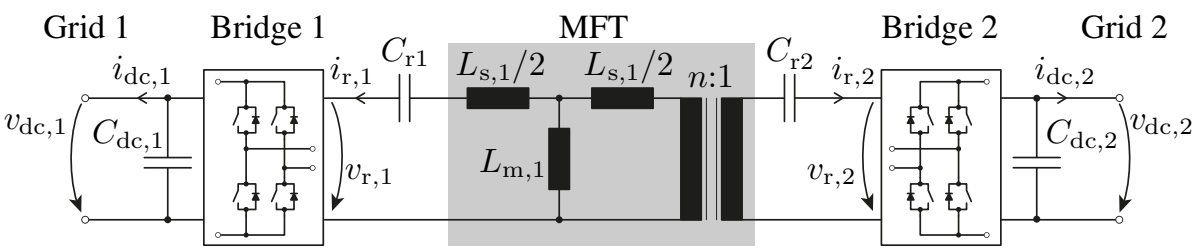

Fig. 1. Investigated dc transformer based on a symmetric LLC resonant converter consisting of two full-bridge inverters (Bridge 1 and Bridge 2) and a resonant tank comprising two resonant capacitors $C_{\mathrm{r} 1}$ and $C_{\mathrm{r} 2}$ and an MFT.

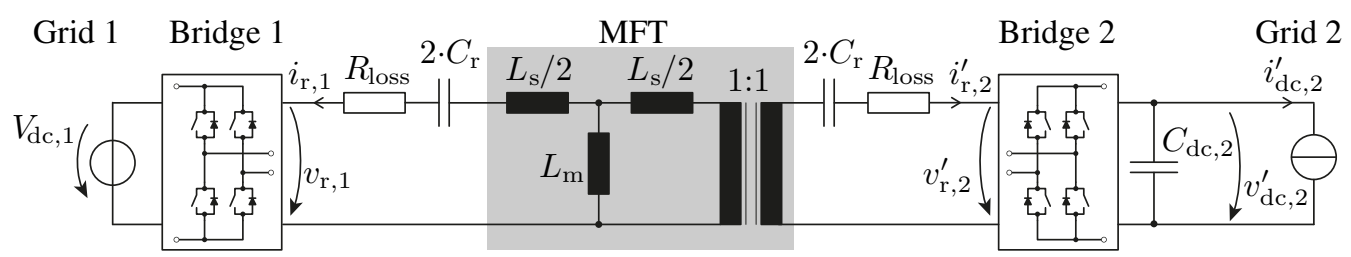

Fig. 2. Model of the dc transformer from Fig. 1 used for the investigations in this paper. All the values are referred to the primary side of the MFT. All the losses are represented by two resistors $R_{\text {loss }}$. Grid 1 is assumed ideally stiff and Grid 2 is used to set the required transferred power via adjustable current source.

The parameters of the MFT do not change $\left(L_{\mathrm{s}}=L_{\mathrm{s}, 1}, L_{\mathrm{m}}=\right.$ $\left.L_{\mathrm{m}, 1}\right)$, since these were already related to the primary side. In the model, the load-dependent losses are represented solely by two resistors $R_{\text {loss }}$ inserted in the resonant tank. Their values are selected to match the required efficiency at the rated power $\eta_{\mathrm{N}}$. The load independent losses, e.g. the switching losses, are neglected in the model.

In the simulations, the voltage of Grid 1 is assumed absolutely stiff, modeled with a constant voltage source $V_{\mathrm{dc}, 1}$, and the power flow is set by the second grid, by adjusting the current of the ideal current source $i_{\mathrm{dc}, 2}^{\prime}$ connected to the $\mathrm{dc}$ link of Bridge 2.

The simulation models were implemented in Mathworks Simulink using Plexim PLECS toolbox to model their electrical part according to Fig. 2. The ABS method is implemented in the native Simulink environment.

\section{CONVERTER Design}

In this section, the impact of the converter design on the voltage characteristics is explored with the goal of deriving good design practices. To provide as generalized results as possible, the study results are provided in p.u. values related to the rated power $P_{\mathrm{N}}$, the switching frequency $f_{\mathrm{s}}$ of the converter, and the rated voltage of Grid $1 V_{\mathrm{dc}, 1}$.

In the following design study, the peak rated magnetizing current $\hat{i}_{L \mathrm{~m}}$ is selected to be $20 \%$ of the rated dc current $I_{\mathrm{dc}, 1}$ :

$$
\hat{i}_{L \mathrm{~m}}=k_{I L \mathrm{~m}} \cdot I_{\mathrm{dc}, 1}=0.2 \cdot I_{\mathrm{dc}, 1}=0.2 \cdot \frac{P_{\mathrm{N}}}{V_{\mathrm{dc}, 1}} .
$$

This value represents $\approx 12 \%$ of the rated peak resonant current and has been selected low enough to guarantee low turn-off losses and high enough to ensure zero-voltage turn on for the switches of the active bridge. Note that this value should not be understood as a universally optimized practice for the design, but rather as a realistic example used to derive general good design practices for LLC-converter-based $\mathrm{dc}$ transformers. In a real design, the characteristics of the applied switches should be considered carefully to determine the most suited peak magnetizing current [27].

The peak rated magnetizing current $\hat{i}_{L \mathrm{~m}}$ determines the required magnetizing inductance $L_{\mathrm{m}}$ of the MFT, using the following approximation from [28]:

$$
\hat{i}_{L \mathrm{~m}}=\frac{V_{\mathrm{dc}, 1}}{4 \cdot L_{\mathrm{m}} \cdot f_{\mathrm{s}}} .
$$

The design values for the stray inductance $L_{\mathrm{S}}$ and the resonant capacitance $C_{\mathrm{r}}$ are determined by the required series resonant frequency

$$
f_{0}=\frac{1}{2 \cdot \pi \cdot \sqrt{L_{\mathrm{s}} \cdot C_{\mathrm{r}}}}
$$

and the required series impedance

$$
Z_{0}=\sqrt{\frac{L_{\mathrm{s}}}{C_{\mathrm{r}}}}
$$

of the resonant tank. For generality, the resonant frequency is further related to the switching frequency: $f_{s} / f_{0}$, and the impedance $Z_{0}$ is related to the rated power and the rated voltage via the quality factor of the equivalent circuit at the rated power

$$
Q_{\mathrm{N}}=\frac{Z_{0}}{R_{\mathrm{ac}}}=\frac{Z_{0}}{\frac{8}{\pi^{2}} \cdot \frac{V_{\mathrm{dc}, 1}^{2}}{P_{\mathrm{N}}}},
$$

usually applied in the literature (e.g., [28]).

To study the voltage characteristic, several hundreds of simulations have been carried out, varying the transmitted power, the quality factor at rated power $Q_{\mathrm{N}}$, the relative switching frequency $f_{\mathrm{s}} / f_{0}$, and the efficiency at the rated power $\eta_{\mathrm{N}}$. In each simulation, the voltage gain

$$
G=\frac{v_{\mathrm{dc}, 2}^{\prime}}{v_{\mathrm{dc}, 1}}=\frac{v_{\mathrm{dc}, 2} \cdot n}{v_{\mathrm{dc}, 1}}
$$

is evaluated at the steady state operation, leading to results shown in Fig. 3. Note that in this narrow range of studied operating points, the first-harmonic analysis, typically used for 

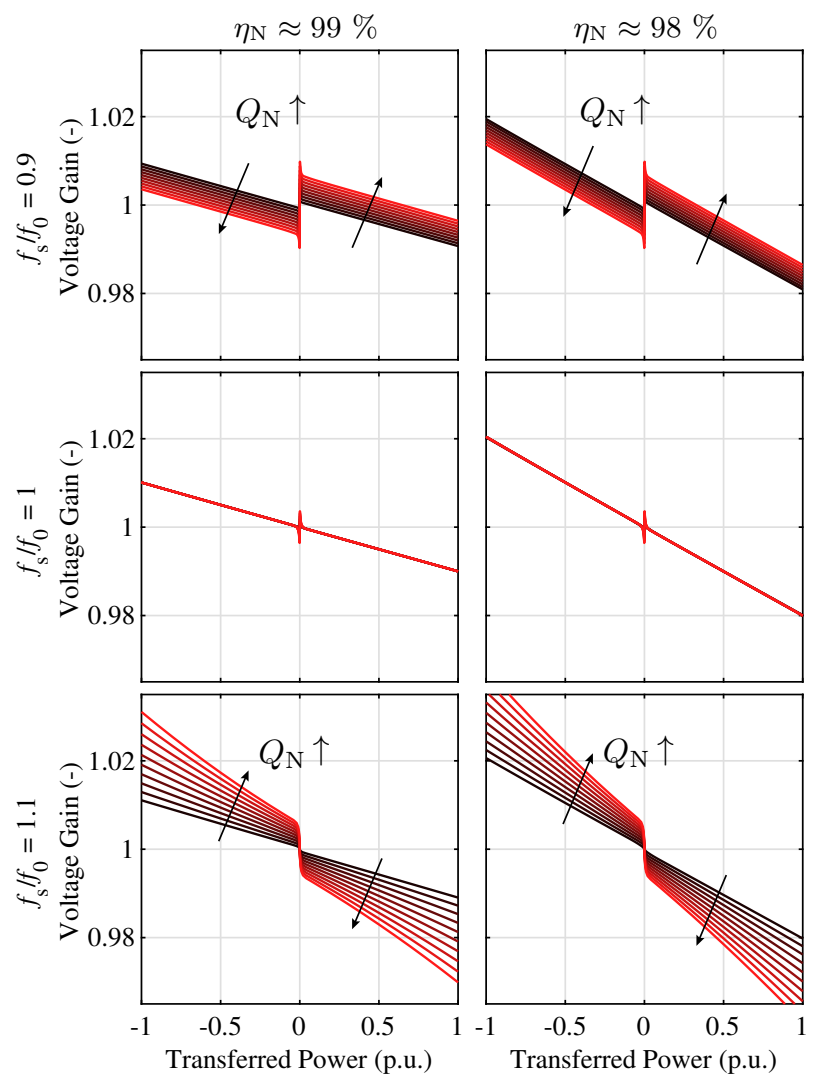

Fig. 3. The dependence of the voltage gain $G$ on the power transferred from Grid 1 to Grid 2 related to the rated power for different factors $Q_{\mathrm{N}}$ varying from 0.05 to 0.5 (in the direction of arrows). The graphs show results for three different relative switching frequencies $f_{\mathrm{s}} / f_{0}$ and two efficiencies at the rated power $\eta_{\mathrm{N}}$. For positive powers, Bridge 1 is actively switching, for negative powers, Bridge 2 is active. The figure demonstrates that the best behavior is achieved when the dc transformer is operated at the resonant frequency.

LLC converter analysis, is too inaccurate (up to $3 \%$ error in the studied range reported in [8], while the expected voltagegain variation in this range is only $\pm 2 \%$ ) and thus, the timedomain simulations have to be used.

Fig. 3 shows that for the operation exactly at the resonant frequency $\left(f_{\mathrm{s}} / f_{0}=1\right.$; middle row), the converter statically behaves as a resistor with losses that correspond to the converter load-dependent losses (voltage gain drop of 0.01 at the rated power for $\eta_{\mathrm{N}}=99 \%$ and 0.02 for $\eta_{\mathrm{N}}=98 \%$ ) independent of the rated quality factor $Q_{\mathrm{N}}$. This is a very welcome behavior, since it guarantees the optimum efficiency power flow in large scale dc grids. The discontinuity observable near zero power is caused by the discontinuous current operation appearing at low tank currents due to the parasitic resistances $R_{\text {loss }}$.

The operation of the converter below the resonant frequency $\left(f_{\mathrm{s}} / f_{0}=0.9\right.$; upper row of Fig. 3 ) shows similar behavior, however, an offset is added to the voltage gain curve depending on the sign of the transferred power and the factor $Q_{\mathrm{N}}$. Generally, the higher the rated quality factor $Q_{\mathrm{N}}$, the further is the characteristic from the ideal "resistor-like" and a smooth power transition becomes less feasible.

Similar observations can be made for the operation above the resonant frequency $\left(f_{\mathrm{s}} / f_{0}=1.1\right.$; last row of Fig. 3$)$ but the offset has a different sign and increasing the $Q_{\mathrm{N}}$ factor

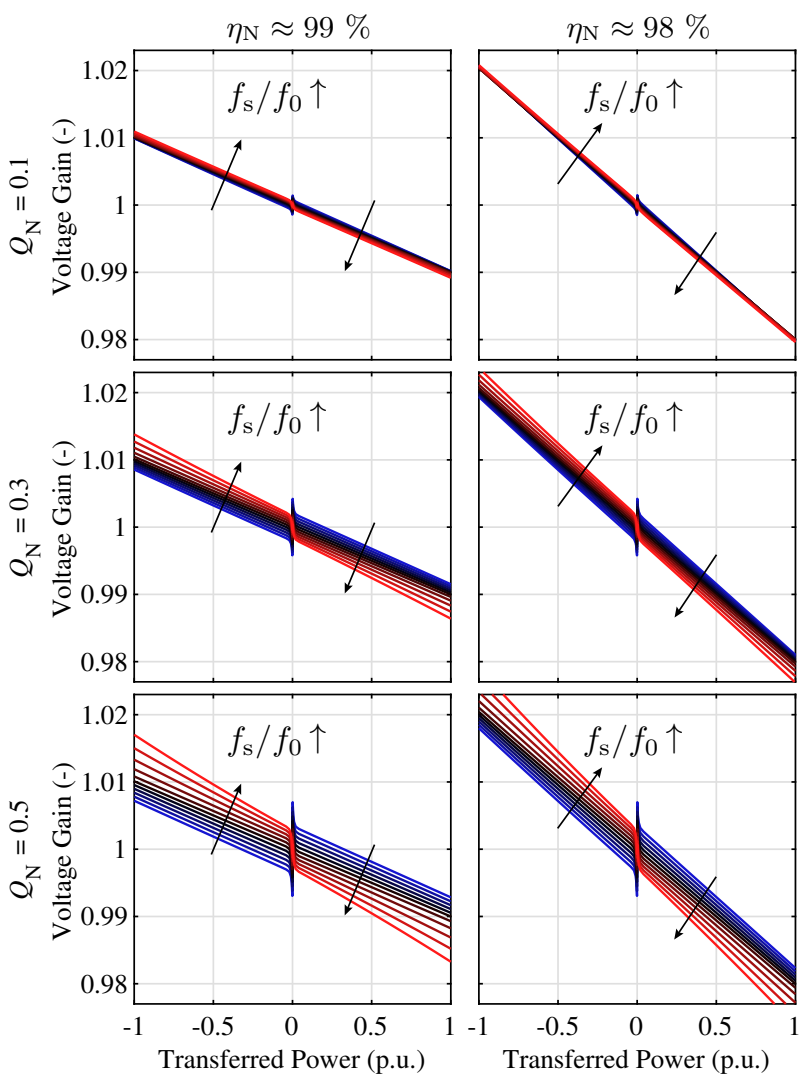

Fig. 4. The dependence of the voltage gain $G$ on the power transferred from Grid 1 to Grid 2 related to the rated power for different relative switching frequencies $f_{\mathrm{s}} / f_{0}$ varying from 0.95 to 1.05 (in the direction of arrows). The graphs show results for three different factors $Q_{\mathrm{N}}$ and two efficiencies at the rated power $\eta_{\mathrm{N}}$. For positive powers, Bridge 1 is actively switching, for negative powers, Bridge 2 is active. The figure demonstrates that the impact of resonant-frequency variation is lower for dc transformers with low $Q_{\mathrm{N}}$.

impacts the slope of the curves as well.

In summary, the best behavior of the converter can be achieved independently of the $Q_{\mathrm{N}}$ factor when the LLC converter is operated at the resonant frequency. Hence, this is the desired operation point of the dc transformer.

Nevertheless, in hardware design, a switching frequency value might be selected slightly lower than the resonant frequency to ensure a constant turn-off current. Moreover, the parameters of the resonant tank are generally subject to manufacturing tolerances and they change slightly during the operation due to the temperature variation and aging. Therefore, a close attention needs to be paid to the impact of the relative switching frequencies $f_{\mathrm{s}} / f_{0}$ on the voltage gain.

In Fig. 4, simulation results for six different converters designed for $Q_{\mathrm{N}} \in\{0.1,0.3,0.5\}$ show the impact of relative switching frequency variation in the range $f_{\mathrm{s}} / f_{0} \in$ $[0.95,1.05]$. This range can be attributed either to an estimation error of the resonant frequency of up to $5 \%$ or to an up to $10 \%$ variation of the stray inductances (or the resonant capacitances) during the dc transformer operation due to, e.g., temperature changes. Fig. 4 clearly shows that the variation of the switching to resonant frequency ratio has less significant impact for lower rated quality factors $Q_{\mathrm{N}}$. Hence, if the rated quality factor $Q_{\mathrm{N}}$ is selected low enough, there is only a minor 
impact of the parameter variation in the resonant tank $\left(C_{\mathrm{r}}\right.$ and $L_{\mathrm{S}}$ ) on the static loading characteristic of the dc transformer, since such variation of parameters slightly modifies both $Q_{\mathrm{N}}$ and $f_{0}$ (both $Q_{\mathrm{N}}$ and $f_{0}$ depend on the square root of the resonant tank parameters $C_{\mathrm{r}}$ and $L_{\mathrm{s}}$ ). In conclusion, the good design practice is to keep the rated quality factor $Q_{\mathrm{N}}$ as low as possible. ${ }^{1}$

A typical constraint for the lowest value of $Q_{\mathrm{N}}$ is the feasible construction of the MFT. The desired low values of $Q_{\mathrm{N}}$ are achieved for low values of $Z_{0}$ which basically means that the stray inductance of the transformer $L_{\mathrm{S}}$ should be minimized [see (9) and (8)].

For the simulations of the proposed method in Section V, the rated quality factor $Q_{\mathrm{N}}=0.1$ is selected. Based on the current experiences, this value is feasible and results in parameters of the resonant tank that are possible to realize as integrated inductances of the MFT [29].

\section{Proposed Active Bridge Switchover Method FOR POWER REVERSAL}

Since the dc transformer is operated in the open loop with a constant switching frequency, it does not obtain any external power setpoint and its operation direction (i.e., which bridge should be actively switched) needs to be decided internally.

The proposed method for deciding on the operation direction is based on observing the power transferred between the dc-links of the converter over the resonant tank. The idea is that once the transferred power drops below a certain threshold, the currently active bridge is deactivated and the other bridge becomes active. This guarantees that the converter operates in the correct power direction when the absolute value of the power is above the defined threshold.

When the required power is below the defined threshold, the bridges are activated alternately, delivering the required power flow as a long-period mean value. Another consequence is a light, and likely acceptable, oscillation of the voltage gain at such low powers.

The most efficient way to observe the resonant-tank power transfer is indirect by observing the peak values $\hat{i}_{\mathrm{r}, 1}$ and $\hat{i}_{\mathrm{r}, 2}$ of the resonant-tank currents $i_{\mathrm{r}, 1}$ and $i_{\mathrm{r}, 2}$, respectively. Since the current on the side of the currently active bridge contains the component of the magnetizing current as well, that does not transfer any power, the tank current on the rectifier side $\left(i_{\mathrm{r}, 1}\right.$ or $i_{\mathrm{r}, 2}$, depending on the currently active bridge) is utilized to estimate whether the power threshold has been crossed. This strategy can be translated into the finite-state machine (FSM) scheme displayed in Fig. 5.

As Fig. 5 shows, either Bridge 1 is active (and Bridge 2 functions as a passive rectifier), or the Bridge 2 is active (and Bridge 1 functions as a rectifier). The transition between these two states is decided one time each switching period $1 / f_{\mathrm{s}}$ depending on the absolute value of the peak resonant-tank current on the rectifier side $\left(\left|\hat{i}_{\mathrm{r}, 1}\right|\right.$ when Bridge 2 is active and

\footnotetext{
${ }^{1}$ A similar observation has been made during design processes in [13], [16]
} for the simple goal of stiff voltage ratio requirement.

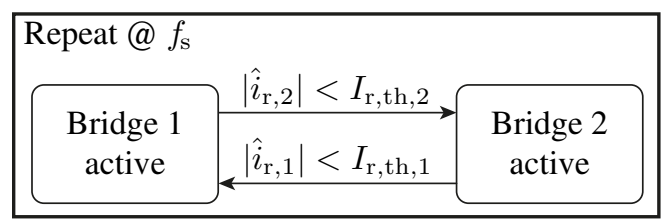

Fig. 5. Finite-state machine of the proposed method.

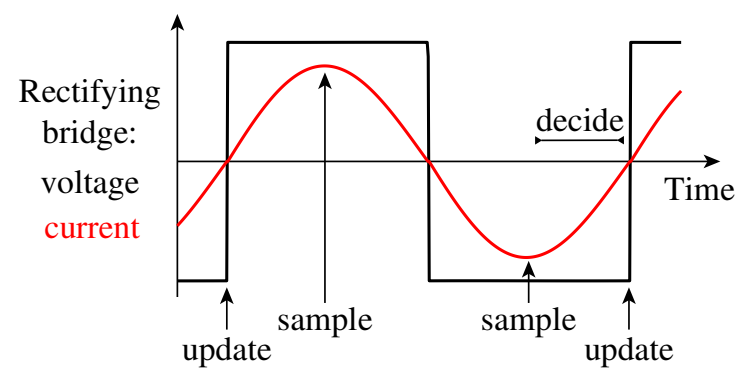

Fig. 6. An example demonstrating the timing of the sampling (labeled by arrows with "sample"), time available for the decision on the operation direction (labeled as "decide") and the time at which the operation direction is updated (labeled by arrows with "update").

$\left|\hat{i}_{\mathrm{r}, 2}\right|$ when Bridge 1 is active). The corresponding threshold values

$$
\begin{aligned}
I_{\mathrm{r}, \mathrm{th}, 1} & =I_{\mathrm{r}, \mathrm{th}} \\
I_{\mathrm{r}, \mathrm{th}, 2} & =n \cdot I_{\mathrm{r}, \mathrm{th}}
\end{aligned}
$$

are set to ensure that the offsets of the current sensors cannot cause an assumption of a non-existing power flow. This can be achieved by selecting the threshold value $I_{\mathrm{r}, \text { th }}$ with a sufficient margin from the worst-case offset and the noise of the current sensors and the utilized ADCs. In this paper, the threshold value is set to $5 \%$ of the rated dc current $I_{\mathrm{dc}, 1}$ (this corresponds to $\approx 3 \%$ of the rated peak resonant current), which should generally provide enough margin for the typical current-sensor offsets.

With this method, only one value captured at each resonant peak of the corresponding resonant current is sufficient for the operation-direction decision. It only has to be ensured that the offset value of the current sensors is below the selected threshold value. The method is not particularly sensitive to gain errors, since these only virtually change the threshold value and thus increase or decrease the power range at which the alternating operation is present. Furthermore, not capturing the measured current value exactly at the peak can be viewed as a small gain error, and thus it is not critical either.

There are several options to implement the proposed ABS method in practice. It can be realized purely in hardware, applying an analog comparator and a resettable register to evaluate if the threshold was hit within the period. Alternatively, the peak currents can be estimated by capturing their value at one fourth and three fourths of the switching period $1 / f_{\mathrm{s}}$ (the latter is theoretically sufficient, since it contains a more up-to-date information). A timing diagram is displayed in Fig. 6, showing the sampling times (labeled as "sample"), the time for processing the FSM ("decide"), and the time point at which the operation direction is updated ("update"). 
TABLE I

SiMULATION PARAMETERS

\begin{tabular}{lcc}
\hline \hline Rated Power & $P_{\mathrm{N}}$ & $10 \mathrm{MW}$ \\
Rated Voltage Grid 1 & $V_{\mathrm{dc}, 1}$ & $5 \mathrm{kV}$ \\
Rated Voltage Grid 2 & $V_{\mathrm{dc}, 2}$ & $10 \mathrm{kV}$ \\
Switching Frequency & $f_{\mathrm{s}}$ & $5 \mathrm{kHz}$ \\
Efficiency at Rated Power & $\eta_{\mathrm{N}}$ & $99 \%$ \\
\hline Threshold Current & $I_{\mathrm{r}, \mathrm{th}}$ & $100 \mathrm{~A}$ \\
Rated Quality Factor & $Q_{\mathrm{N}}$ & 0.1 \\
Resonant Frequency & $f_{0}$ & $5 \mathrm{kHz}$ \\
\hline Transformer Ratio & $n$ & $1 / 2$ \\
Stray Inductance & $L_{\mathrm{s}, 1}$ & $6.45 \mu \mathrm{H}$ \\
Magnetizing Inductance & $L_{\mathrm{m}, 1}$ & $625 \mu \mathrm{H}$ \\
Resonant Capacitance 1 & $C_{\mathrm{r}, 1}$ & $314.16 \mu \mathrm{F}$ \\
Resonant Capacitance 2 & $C_{\mathrm{r}, 2}$ & $78.54 \mu \mathrm{F}$ \\
Equivalent Loss Resistance (primary side) & $R_{\mathrm{loss}, 1}$ & $10.13 \mathrm{~m} \Omega$ \\
Equivalent Loss Resistance (secondary side) & $R_{\mathrm{loss}, 2}$ & $40.53 \mathrm{~m} \Omega$ \\
DC-link Capacitance & $C_{\mathrm{dc}, 2}$ & $8 \mathrm{mF}$ \\
\hline
\end{tabular}

Note that the direction could be theoretically updated two times per period (at both rising and falling edges) to improve the response time of the dc transformer. However, this practice can excite the weakly damped "secondary" resonant circuit composed of the magnetizing inductor and the resonant capacitors. Therefore, it is recommended to update the operation direction only at one of the edges (e.g., rising edge as shown in Fig. 6).

In practical applications, the sensor offset can be corrected from drifting, since the measured current is an ac current (e.g., by using the mean value of both sampled values). Furthermore, some measurement redundancy can be achieved by removing the predicted magnetizing current value from the measured current of the active bridge. If required, the peak resonant current can be also estimated by measuring the change of the corresponding resonant capacitor voltage at the rising and falling edges. Then, the switchover thresholds are set according to the voltage sensor noise to ensure that the required operation direction is estimated correctly.

Moreover, it is important to note that the proposed ABS method has a positive effect on the discontinuity near zero power in the characteristics shown in Fig. 3 and Fig. 4. This is because the FSM changes the active bridge before the nonlinear discontinuous-current operation of the LLC converter begins. As a consequence, it should be ensured in practical applications that the selected value $I_{\mathrm{r}, \mathrm{th}}$ is above this nonlinear region. In practice, the sufficient value can be estimated either via simulations or in experiments. The absence of this non-linear discontinuous region with the proposed method will be validated in the next section.

\section{Simulations}

To demonstrate the properties of the proposed ABS method, a series of time-domain simulations has been carried out.

To show the performance at different power profiles while omitting the dynamics of the connected dc grids, the combination of the constant grid voltage $V_{\mathrm{dc}, 1}$ at Grid 1 and adjustable grid current $i_{\mathrm{dc}, 2}$ that sets power at Grid 2 is applied in these simulations as well.

For the following performance studies, two power profiles are applied:

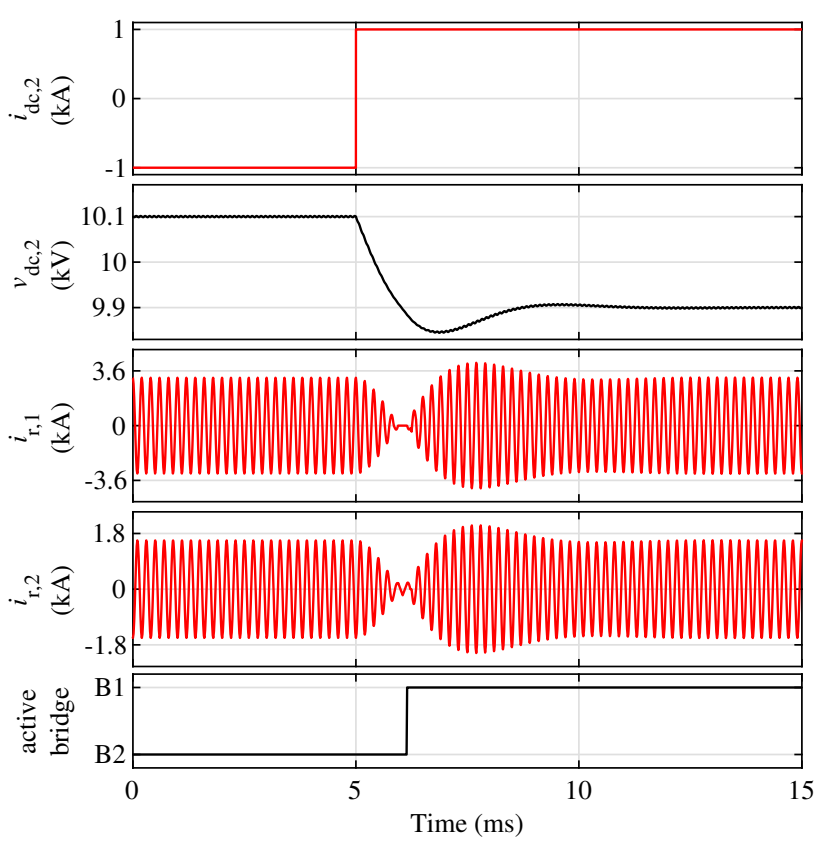

Fig. 7. Operation of the dc transformer employing the proposed ABS method at a fast power transition from negative rated power to positive rated power.

- a step from the negative rated power to the positive rated power to demonstrate the dynamics of the worst case, and

- slowly increasing power from the negative rated value to the positive rated value, changing the operating point quasi-statically, thus showing a wide range of operating points.

\section{A. DC Transformer Parameters}

The parameters for the simulated dc transformer are listed in Table I. These parameters represent a $10 \mathrm{MW} 5 \mathrm{kV} / 10 \mathrm{kV}$ $\mathrm{dc}$ transformer. Since the simulated example is a high-power medium-voltage application, the operating frequency has been chosen relatively low at $5 \mathrm{kHz}$. The rated efficiency is selected to be $99 \%$, since low losses represent the worst-case scenario in terms of damping (and thus converter stability). As mentioned before, the threshold current is $5 \%$ of the rated primary $\mathrm{dc}$ current and the required turn-off current is $20 \%$ of the same. The quality factor was selected to be $Q_{\mathrm{N}}=0.1$ and the converter is operated at the resonant frequency $\left(f_{\mathrm{s}} / f_{0}=1\right)$.

The rest of the parameters can be determined according to the design process described in Section III. Note that the selected parameters represent only an example with the purpose to demonstrate the novel ABS method. The lowest possible quality factor $Q_{\mathrm{N}}$ is subject to lowest achievable stray inductances of the MFT. Nevertheless, these can be determined only via a detailed MFT design process such as that in [30] and is out of scope of this paper. The dc-link capacitance was selected high enough to allow for well-damped behavior during the load steps. The detailed design of the dc-link capacitances will be addressed in the future research. 


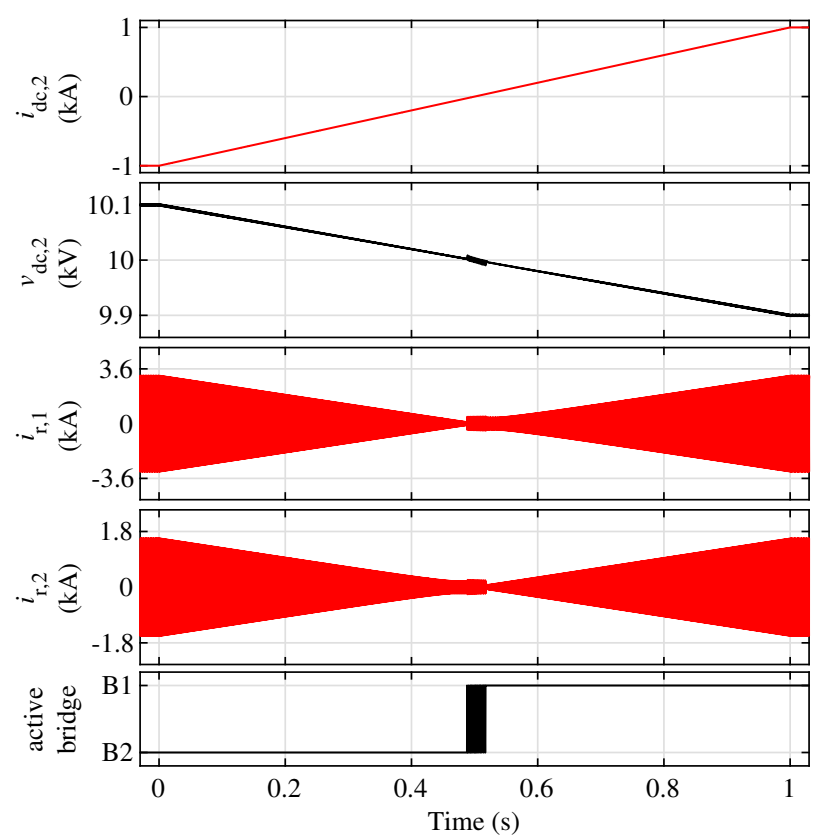

(a)

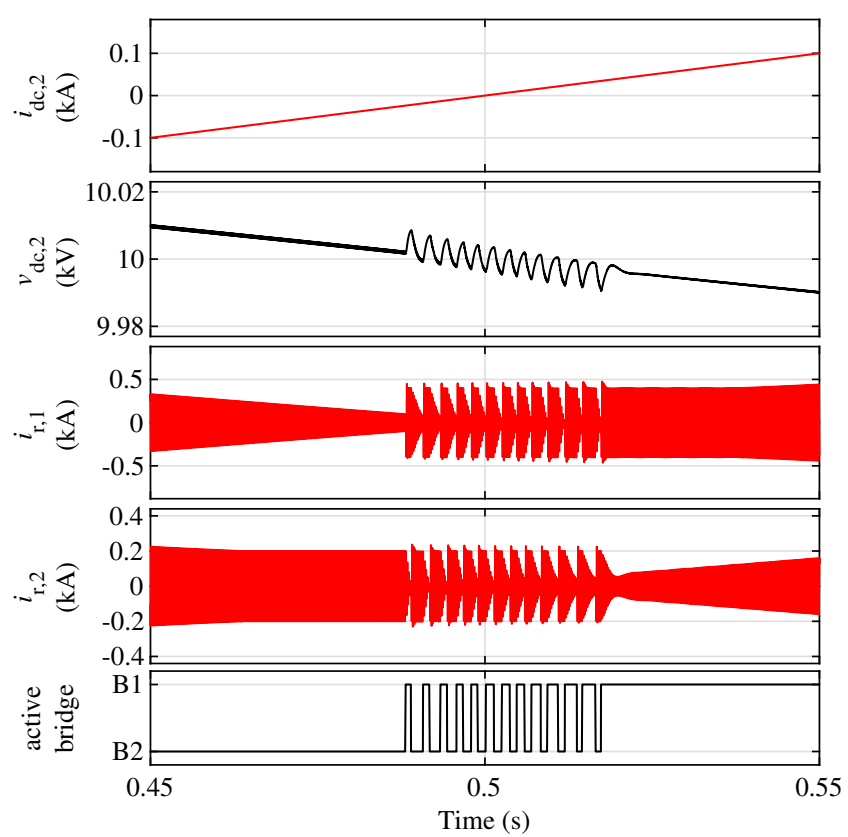

(b)

Fig. 8. Operation of the dc transformer employing the proposed ABS method at a slow power transition from negative rated power to positive rated power: (a) full time scale, (b) detail showing the transition near zero power.

\section{B. Performance at Worst-Case Load Step}

Fig. 7 shows the performance of the converter at the worstcase load step from the rated negative power $-P_{\mathrm{N}}$ to the rated positive power $P_{\mathrm{N}}$ applied at a time instance $t_{\mathrm{step}}=5 \mathrm{~ms}$. As mentioned above, the power is set by the current of Grid 2 $i_{\mathrm{dc}, 2}$.

At the time $t=0$, the converter operates in the steadystate. Bridge 2 (B2) is active and Bridge 1 (B1) operates as a passive rectifier. The voltage at the secondary dc-link $v_{\mathrm{dc}, 2}$ has settled at a value according to the gain characteristic (since the primary dc-link voltage is constant $\left.V_{\mathrm{dc}, 1}\right)$.

Once the power step is applied at the time $t_{\text {step }}=5 \mathrm{~ms}$, it can be seen that the converter initially continues to deliver the power according to the voltage gain characteristic. The dc-link capacitor $C_{\mathrm{dc}, 2}$ is hence being discharged from both sides (Bridge 2 and Grid 2) and its voltage drops rapidly. Consequently, the voltage gain approaches $G=1$ and the transferred power through the resonant tank (resonant currents $i_{\mathrm{r}, 1}$ and $i_{\mathrm{r}, 2}$ ) decreases. Only after the dc-link voltage crossed $v_{\mathrm{dc}, 2} \approx 10 \mathrm{kV}$ (which corresponds to voltage gain $G=1$ ), the current is led to zero with a short delay, typical for LLC converters [31], and the threshold on the rectifier side (Bridge 1 ) is crossed. Then, Bridge 1 is activated and the currents in the resonant tank increase until the steady state at the positive rated power is reached.

In summary, the power reversal at the rapid load step is performed smoothly and stably.

\section{Performance at Slow Power Sweep}

In the second simulation, plotted in Fig. 8a, the power is swept slowly from the negative rated power to the positive rated power in a linear ramp (visible at current $i_{\mathrm{dc}, 2}$ ). Since the power sweep is very slow, the curves can be represented as quasi-static, showing a wide range of operating points. It can be seen that the voltage of Grid 2 (and thus voltage gain) decreases linearly with the increased transferred power. Furthermore, it can be seen that the peak values of resonanttank currents $i_{\mathrm{r}, 1}$ and $i_{\mathrm{r}, 2}$ follow the trend of the transferred power. When the transferred power is above the threshold and positive $\left(i_{\mathrm{dc}, 2}\right.$ is positive), Bridge 1 is active. When the power is above the threshold and negative, Bridge 2 is active. When the transferred power is near zero and thus below the threshold, the active bridge is alternated between Bridge 1 and Bridge 2 . To observe this phenomenon closely, Fig. 8b shows a detail of Fig. 8a for transferred powers near zero.

As Fig. 8b shows, once the transferred power is below the defined threshold, the alternation between the bridges takes place. It can be seen that the currently active bridge conducts mainly the magnetizing current (visible as almost constant current envelope of $400 \mathrm{~A}$ in $i_{\mathrm{r}, 1}$ and $200 \mathrm{~A}$ in $i_{\mathrm{r}, 2}$ ) and the currents of the rectifying bridge decay until the next switchover of active bridges is initiated. The ratio between the active times of both bridges follows naturally the transmitted power. It can be seen that the alternation of bridges causes a ripple (an oscillation) at the dc-the link voltage of Grid $2 v_{\mathrm{dc}, 2}$ which can be associated to a small oscillation of the voltage gain. Despite this ripple, the underlying waveform of the Grid 2 voltage follows the desired voltage gain characteristic that corresponds to the load-dependent converter losses.

Another point that can be observed in Fig. 8 is that the voltage gain discontinuity at zero power, observable in Fig. 3 and Fig. 4, does not occur in the dynamic simulation. As mentioned before, this is because the operation direction is switched-over at higher currents than those at which this non- 


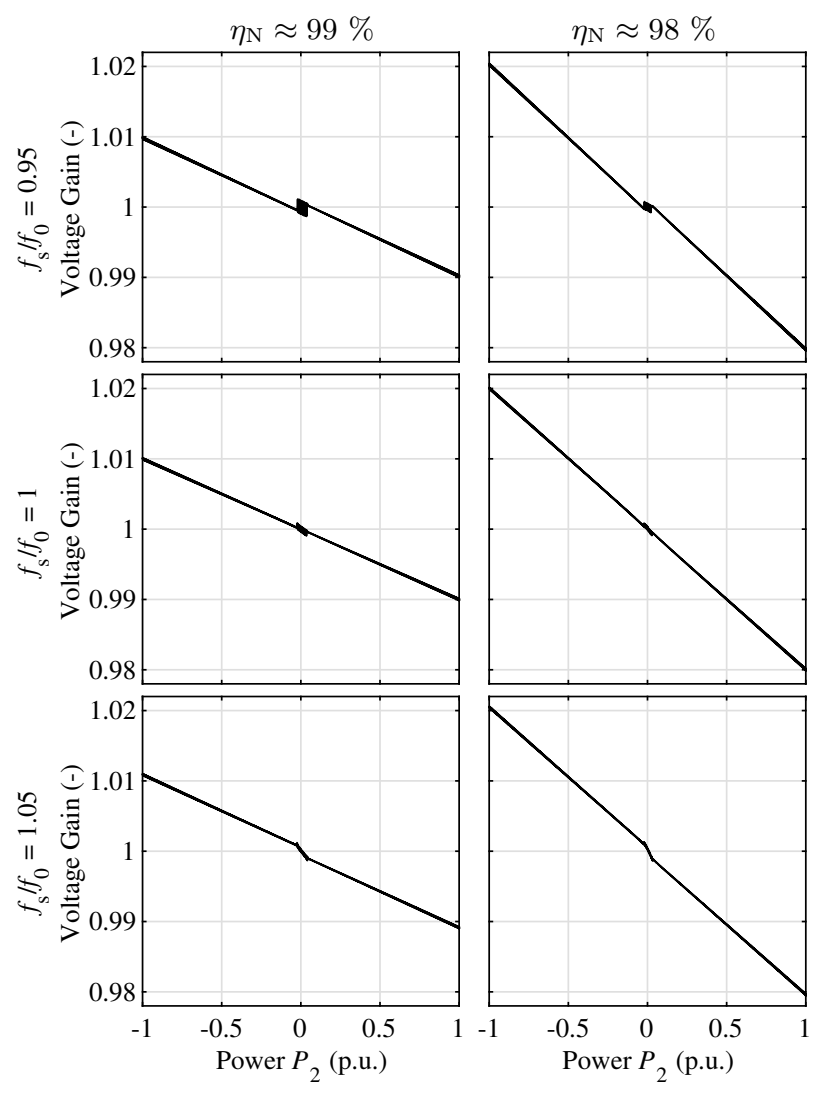

Fig. 9. Simulated quasi-static dependence of the voltage gain $G$ on the power transferred to Grid $2 P_{2}$ related to the rated power for different switching frequencies.

linear behavior occurs.

\section{Parameter Variation}

To evaluate the influence of the parameter variation, the slow-power-sweep simulation has been carried out for the same converter with varied switching frequency (mismatching the resonance frequency) and additionally for the cases with decreased efficiency $\left(\eta_{\mathrm{N}}=98 \%\right)$. The voltage $v_{\mathrm{dc}, 2}$ and the current $i_{\mathrm{dc}, 2}$ obtained from the simulations were utilized to determine the Grid 2 power $P_{2}$ versus voltage gain $G$ quasistatic characteristics displayed in Fig. 9.

In general, Fig. 9 confirms that the dc transformer is capable of very smooth and almost linear power reversal even at relatively wide range of mismatched switching frequencies. Furthermore, this indicates that the method is not expected to be sensitive to the slight variations of the inductances during the operation (since these can be represented by the even slighter switching frequency variation).

Comparing the simulated quasi-static characteristics of the $\mathrm{dc}$ transformer employing the proposed power direction estimation method (Fig. 9) to the static characteristics (Fig. 3), it can be recognized that the quasi-static operation follows the trend of the static operation, except for the region near zero power where the behavior is linearized and thus, improved.

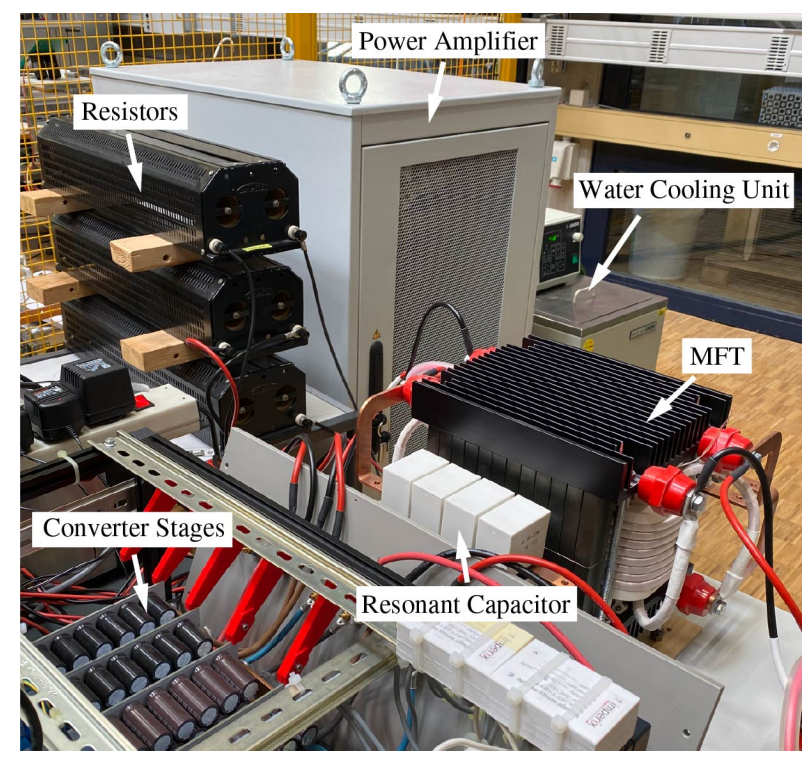

Fig. 10. Photo of the low-voltage low-power dc transformer prototype.

TABLE II

EXPERIMENTAL PROTOTYPE PARAMETERS

\begin{tabular}{lcc}
\hline \hline Rated Power & $P_{\mathrm{N}}$ & $5 \mathrm{~kW}$ \\
Rated Voltage Grid 1 & $V_{\mathrm{dc}, 1}$ & $200 \mathrm{~V}$ \\
Rated Voltage Grid 2 & $V_{\mathrm{dc}, 2}$ & $200 \mathrm{~V}$ \\
Switching Frequency & $f_{\mathrm{s}}$ & $10.8 \mathrm{kHz}$ \\
Rel. Load-Dependent Losses & $\epsilon_{\mathrm{N}}$ & $\approx 3 \%$ \\
\hline Threshold Current & $I_{\mathrm{r}, \mathrm{th}}$ & $1.25 \mathrm{~A}$ \\
Rated Quality Factor & $Q_{\mathrm{N}}$ & 0.12 \\
Resonant Frequency & $f_{0}$ & $10.8 \mathrm{kHz}$ \\
\hline Transformer Ratio & $n$ & 1 \\
Stray Inductance & $L_{\mathrm{s}, 1}$ & $11.6 \mu \mathrm{H}$ \\
Magnetizing Inductance & $L_{\mathrm{m}, 1}$ & $750 \mu \mathrm{H}$ \\
Resonant Capacitance 1 & $C_{\mathrm{r}, 1}$ & $37.5 \mu \mathrm{F}$ \\
Resonant Capacitance 2 & $C_{\mathrm{r}, 2}$ & $37.5 \mu \mathrm{F}$ \\
DC-link Capacitance 1 & $C_{\mathrm{dc}, 1}$ & $520 \mu \mathrm{F}$ \\
DC-link Capacitance 2 & $C_{\mathrm{dc}, 2}$ & $520 \mu \mathrm{F}$ \\
\hline \hline
\end{tabular}

\section{EXPERIMENTAL VALIDATION}

\section{A. Low-Voltage Prototype}

To validate the practical feasibility of the proposed ABS method, a low-voltage low-power prototype, pictured in Fig. 10, was built. The prototype is composed of two IGBT full bridges, each consisting of two Imperix PEB 8032 half bridge modules rated at $800 \mathrm{~V}$ and $32 \mathrm{~A}$. The rated module current limits the rated dc current to $\approx 25 \mathrm{~A}$. The resonant tank consists of a $100 \mathrm{~kW} \mathrm{1:1} \mathrm{MFT} \mathrm{with} \mathrm{integrated} \mathrm{resonant}$ capacitors that was developed in [30]. The ABS method is implemented on the Imperix Boombox fast prototyping control platform.

To keep the magnetizing current of the transformer reasonably low during the operation, the rated voltage $V_{\mathrm{dc}, 1}$ was set to $200 \mathrm{~V}$ which leads to the relative peak magnetizing current of $k_{I L \mathrm{~m}}=0.25$.

The connected dc grids are represented by the four phases of Regatron TC.ACS.50.528.72 switched power amplifier. Since the power amplifier operates as a voltage source, a $6.8 \Omega$ resistor (constructed as three series resistors in Fig. 10) was connected in series to the amplifier on the Grid 2 side. 

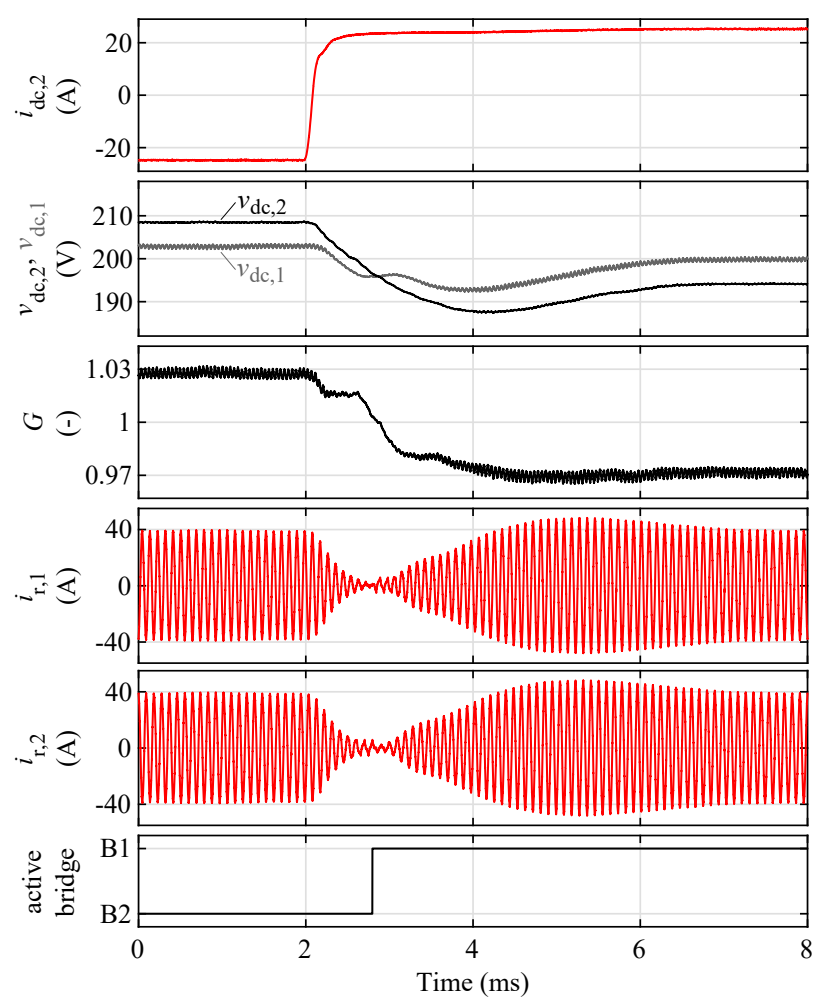

Fig. 11. Experimental waveforms showing the response of the dc transformer prototype employing the ABS method to the worst-case power step at Grid 2.

By controlling the voltage drop over this resistor through the amplifier, the Grid 2 current can be set with sufficient precision.

The parameters of the converter prototype are summarized in Table II. Note that the value of the stray inductance $L_{\mathrm{s}, 1}$ is increased compared to the value from [30] to account for the stray inductance of the cables. Despite clearly different ratings compared to those of the medium-voltage dc transformer from the simulations, it can be observed that the two main design parameters $Q_{\mathrm{N}}$ and $f_{\mathrm{s}} / f_{0}$ of the low-voltage prototype are similar and thus, similar behavior is expected. The rated efficiency was measured to be $96.2 \%$ and the relative loaddependent losses at the rated power are estimated to be $\epsilon_{\mathrm{N}} \approx 3 \%$.

\section{B. Experimental Results}

The investigations of the dc transformer dynamic response to load changes that were carried out in simulations were repeated as experiments to validate the practical feasibility of the proposed ABS method.

The first experiment, which results are in Fig. 11, demonstrates the response of the dc transformer to a worst-case current step from negative rated value to positive rated value at Grid 2. A very similar observations to those for the simulation results from Fig. 7 can be made: The whole transition can be concluded to be smooth and stable. The main difference is that the power amplifier is not capable of holding the voltage of Grid $1 v_{\mathrm{dc}, 1}$ constant during such fast power step and thus, the whole transition is relatively slower.
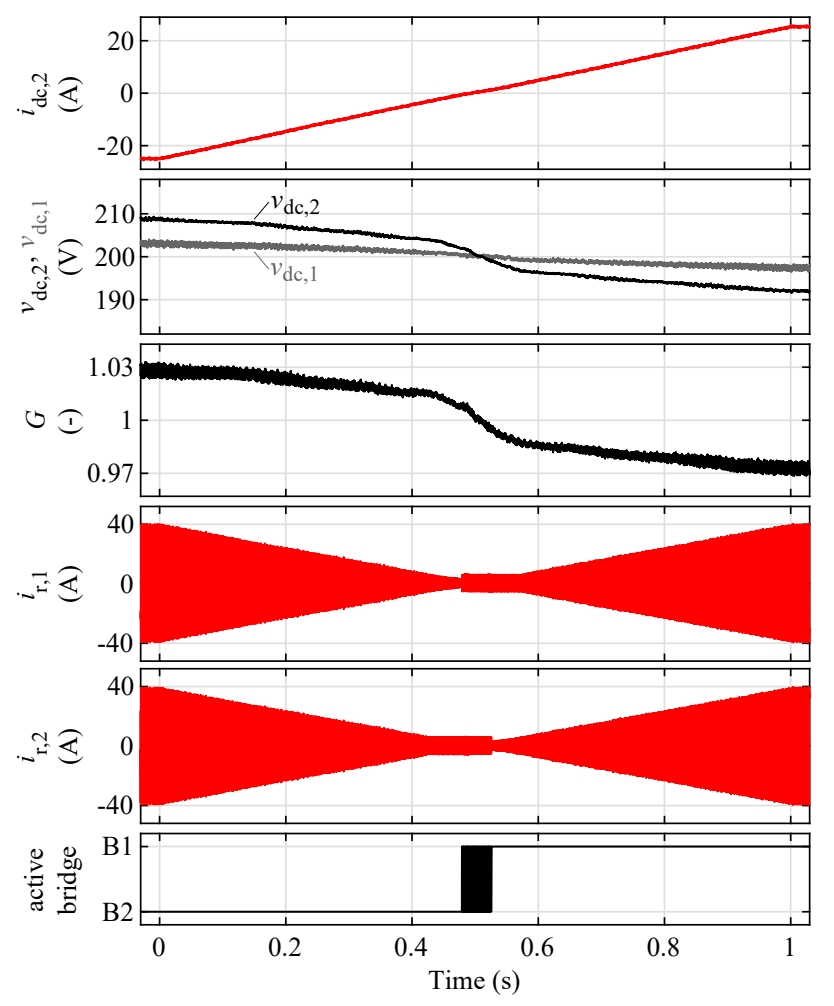

Fig. 12. Experimental waveforms showing the response of the dc transformer prototype employing the ABS method to a slow power ramp at Grid 2.

The second experiment was the application of a slow power ramp to the dc transformer to demonstrate the behavior in large range of operating points. The experimental results in Fig. 12 show a good match to the simulation results from Fig. 8. The operation can be concluded smooth and the alternation of the active bridges at low transferred powers is clearly visible. The main observable difference to the simulation is the altered form of the voltage gain $G$ (in Fig. 8, $G$ can be observed indirectly via $\left.v_{\mathrm{dc}, 2}\right)$. This is caused by the forward threshold voltages of the IGBTs and diodes that were not accounted for in the simulations. As can be seen in Fig. 12, this threshold voltages cause increased losses near zero currents which impact the steepness of the voltage gain curve at this region. A similar effect on the voltage gain was demonstrated in [32] for series resonant converters. Nonetheless, since the voltage gain still "emulates" the voltage drop over the dc transformer that corresponds to its load-dependent losses, this effect is not expected to impact the naturally most efficient power flow in the dc grids.

Finally, Fig. 13 shows the zoomed in waveforms captured at the resonant tank. Fig. 13a demonstrates the typical LLC operation at resonant frequency when rated power is transferred from Grid 1 (Bridge 1 is switching). Fig. 13b demonstrates the operation at no load. Since the direction of the power is not clear (the rectified currents are low), Bridge 1 and Bridge 2 are switched alternately, transferring a small amount of energy back-and-forth between the dc links of the dc transformer. The magnetizing current of the transformer is delivered by either of the bridges. In Fig. 13b, it can be noticed that after the 


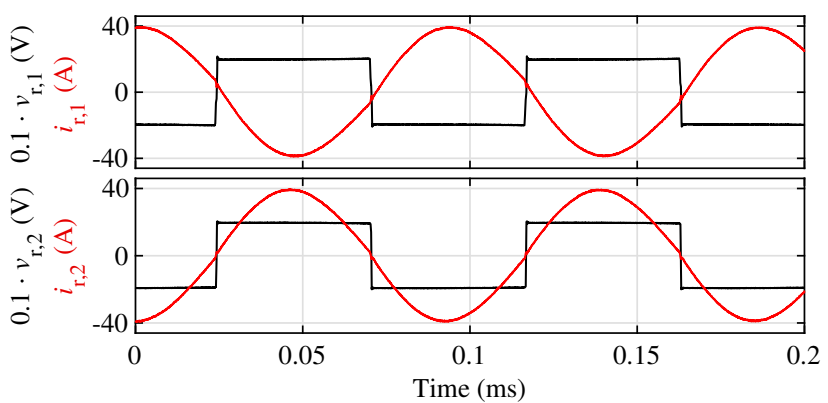

(a)
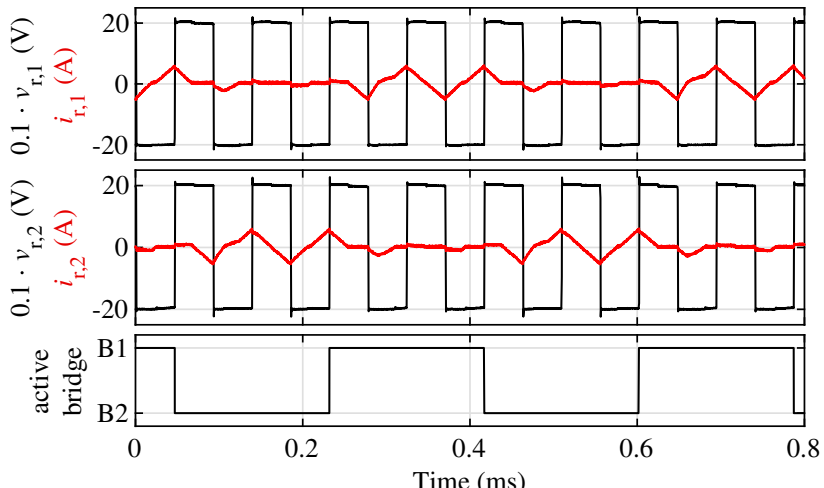

(b)

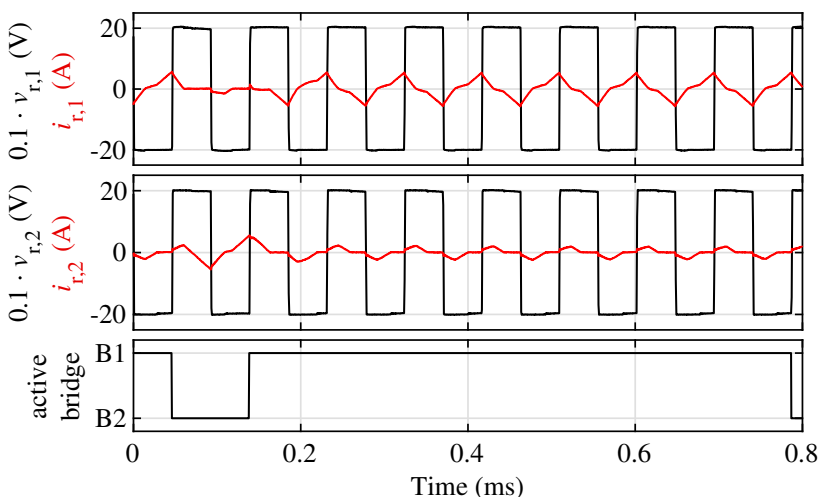

(c)

Fig. 13. Detailed experimental waveforms captured at the resonant tank of the dc transformer prototype: (a) rated power delivered by Grid 1 (Bridge 1 is active) (b) no-load condition (c) light-load condition (3\% of the rated power delivered by Grid 1).

switchover, the magnetizing current continues to be delivered by the previously active bridge. This is because the stray inductances of the transformer do not allow discontinuity of the tank currents. Only after the current reaches zero, the active bridge starts to deliver the magnetizing current. As a consequence, immediately after the switchover of the currently active bridge, its IGBTs are not turned on with ZVS but rather with ZCS. Similar occurrences can be observed in the waveforms for the light-load condition, depicted in Fig. 13c. The power is transferred from Grid 1 to Grid 2 and thus, the Bridge 1 is active for the most of the time. Nevertheless, since the power is low, the current in the resonant tank repeatedly drops bellow the threshold value and the Bridge 2 is activated for a short period of time.

\section{CONCLUSIONS}

This paper studied options for a smooth power direction transition of a dc transformer based on an LLC converter. Within the paper, practices for the design of the resonant tank are derived and a novel ABS method for power reversal is proposed.

The design study concludes that it is advisable to operate the converter at a switching frequency that is reasonably close to the resonant frequency. Furthermore, to ensure a low sensitivity of the voltage gain to the operation frequency, the quality factor of the converter should be as low as possible.

The proposed ABS method for seamless power reversal proved to work smoothly and stably in both simulations and experiments. The method does not need any information from the connected grids and does not employ any closed-loop controllers. The only additional hardware is the two ac-current sensors placed at the resonant tank. However, these sensors do not have to be particularly precise and thus, they are not expected to increase the converter costs significantly.

Compared to the solutions from the literature [12]-[23], the proposed method operates only one bridge at a time and thus, it reduces switching losses and is less sensitive to parameter variation. Hence, the proposed method is expected to be advantageous for LLC converter based dc transformers that employ bipolar semiconductor switches.

The future research will cover the impact of the presented dc transformer on the stability of the connected dc grids and options for the grid and dc-transformer protection. To further confirm the feasibility of the concept, a high-power mediumvoltage demonstrator will be developed.

\section{REFERENCES}

[1] Cigre WG C6.31, "Medium voltage direct current (MVDC) grid feasibility study," CIGRE, pp. 1-127, 2020.

[2] "IEEE Recommended Practice for $1 \mathrm{kV}$ to $35 \mathrm{kV}$ Medium-Voltage DC Power Systems on Ships," IEEE Standard 1709, 2010.

[3] U. Javaid et al., "MVDC marine electrical distribution: Are we ready?" in IECON 2015 - 41st Annual Conference of the IEEE Industrial Electronics Society, 2015, pp. $000823-000828$.

[4] (2020) ANGLE DC project. [Online]. Available: https://www. spenergynetworks.co.uk/pages/angle_dc.aspx

[5] Y. Hu et al., "High-frequency-link current stress optimization of cascaded h-bridges based solid-state transformer with third-order harmonic voltage injection," IEEE Journal of Emerging and Selected Topics in Power Electronics, pp. 1-1, 2020.

[6] R. W. A. A. De Doncker et al., "A three-phase soft-switched high-powerdensity dc/dc converter for high-power applications," IEEE Transactions on Industry Applications, vol. 27, no. 1, pp. 63-73, 1991.

[7] X. Li and A. K. S. Bhat, "Analysis and design of high-frequency isolated dual-bridge series resonant dc/dc converter," IEEE Transactions on Power Electronics, vol. 25, no. 4, pp. 850-862, 2010.

[8] W. Chen et al., "Snubberless bidirectional dc-dc converter with new CLLC resonant tank featuring minimized switching loss," IEEE Transactions on Industrial Electronics, vol. 57, no. 9, pp. 3075-3086, 2010.

[9] J. Jung et al., "Design methodology of bidirectional CLLC resonant converter for high-frequency isolation of dc distribution systems," IEEE Transactions on Power Electronics, vol. 28, no. 4, pp. 1741-1755, 2013.

[10] S. Kenzelmann et al., "Isolated $\mathrm{dc} / \mathrm{dc}$ structure based on modular multilevel converter," IEEE Transactions on Power Electronics, vol. 30, no. 1, pp. 89-98, 2015.

[11] I. A. Gowaid et al., "Quasi two-level operation of modular multilevel converter for use in a high-power dc transformer with dc fault isolation capability," IEEE Transactions on Power Electronics, vol. 30, no. 1, pp 108-123, 2015. 
[12] W. Feng et al., "Pulsewidth locked loop (PWLL) for automatic resonant frequency tracking in LLC dc-dc transformer (LLC -DCX)," IEEE Transactions on Power Electronics, vol. 28, no. 4, pp. 1862-1869, 2013.

[13] K. Tomas-Manez et al., "Unregulated series resonant converter for interlinking dc nanogrids," in 2017 IEEE 12th International Conference on Power Electronics and Drive Systems (PEDS), 2017, pp. 647-654.

[14] C. Zhao et al., "Power electronic traction transformer-medium voltage prototype," IEEE Transactions on Industrial Electronics, vol. 61, no. 7, pp. 3257-3268, 2014.

[15] C. Ma et al. "Si-IGBT versus SiC-MOSFET — an isolated bidirectional resonant LLC dc-dc converter for distributed power systems," in 2015 54th Annual Conference of the Society of Instrument and Control Engineers of Japan (SICE), 2015, pp. 894-899.

[16] J. Huang et al., "Implementation of bidirectional resonant dc transformer in hybrid ac/dc micro-grid," IEEE Transactions on Smart Grid, vol. 10, no. 2, pp. 1532-1542, 2019.

[17] K. Tan et al., "Optimal design methodology of bidirectional LLC resonant $\mathrm{dc} / \mathrm{dc}$ converter for solid state transformer application," in IECON 2014 - 40th Annual Conference of the IEEE Industrial Electronics Society, 2014, pp. 1657-1664.

[18] X. Yu and P. Yeaman, "A new high efficiency isolated bi-directional dc-dc converter for dc-bus and battery-bank interface," in 2014 IEEE Applied Power Electronics Conference and Exposition - APEC 2014, 2014, pp. 879-883.

[19] J. Zhang et al., "An LLC-LC type bidirectional control strategy for an LLC resonant converter in power electronic traction transformer," IEEE Transactions on Industrial Electronics, vol. 65, no. 11, pp. 8595-8604, 2018.

[20] J. Xu et al., "PWM modulation and control strategy for LLC-DCX converter to achieve bidirectional power flow in facing with resonant parameters variation," IEEE Access, vol. 7, pp. 54693-54 704, 2019.

[21] T. Guillod et al., "Active magnetizing current splitting ZVS modulation of a $7 \mathrm{kV} / 400 \mathrm{~V}$ dc transformer," IEEE Transactions on Power Electronics, vol. 35, no. 2, pp. 1293-1305, 2020.

[22] Y. Liao et al., "Single-stage DAB-LLC hybrid bidirectional converter with tight voltage regulation under DCX operation," IEEE Transactions on Industrial Electronics, pp. 1-1, 2020.

[23] W. Mei et al., "A novel bidirectional control strategy for LLC resonan converter in high voltage application," in 2019 IEEE Vehicle Power and Propulsion Conference (VPPC), 2019, pp. 1-5.

[24] J. M. Burdio et al., "Asymmetrical voltage-cancellation control for full-bridge series resonant inverters," IEEE Transactions on Power Electronics, vol. 19, no. 2, pp. 461-469, 2004.

[25] Y. Shen et al., "A bidirectional resonant dc-dc converter suitable for wide voltage gain range," IEEE Transactions on Power Electronics, vol. 33, no. 4, pp. 2957-2975, 2018.

[26] Q. Chen et al., "Soft starting strategy of bidirectional LLC resonant dc-dc transformer based on phase-shift control," in 2014 9th IEEE Conference on Industrial Electronics and Applications, 2014, pp. 318322.

[27] D. Dujic et al., "Characterization of $6.5 \mathrm{kV}$ IGBTs for high-power medium-frequency soft-switched applications," IEEE Transactions on Power Electronics, vol. 29, no. 2, pp. 906-919, 2014.

[28] Bing Lu et al., "Optimal design methodology for LLC resonant converter," in Twenty-First Annual IEEE Applied Power Electronics Conference and Exposition, 2006. APEC '06., 2006, pp. 6 pp.-

[29] M. Mogorovic and D. Dujic, "Analysis of the effectiveness of the series inductor integration into the MFT for SST applications," Proceedings PCIM Europe 2020 (download version), 2020. [Online]. Available: http://infoscience.epfl.ch/record/278527

[30] M. Mogorovic and D. Dujic, " $100 \mathrm{~kW}, 10 \mathrm{kHz}$ medium-frequency transformer design optimization and experimental verification," IEEE Transactions on Power Electronics, vol. 34, no. 2, pp. 1696-1708, 2019

[31] J. E. Huber et al., "Generic derivation of dynamic model for halfcycle DCM series resonant converters," IEEE Transactions on Power Electronics, vol. 33, no. 1, pp. 4-7, 2018.

[32] M. Steiner and H. Reinold, "Medium frequency topology in railway applications," in 2007 European Conference on Power Electronics and Applications, 2007, pp. 1-10.

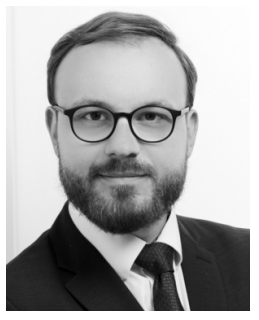

Jakub Kucka (Member, IEEE) received the Bc. and Ing. degrees in electrical engineering from Czech Technical University, Prague, Czech Republic, in 2012 and 2014, respectively, and the Dr.-Ing. (Ph.D.) degree from Leibniz University Hannover, Germany, in 2019 .

From 2014 to 2019, he was a Research Associate with the Institute for Drive Systems and Powe Electronics, Leibniz University Hannover, Germany. Since 2020, he has been a Postdoctoral Researcher with the Power Electronics Laboratory, EPFL, Lausanne, Switzerland. His research interests include modular multilevel converters, converter control and design, and resonant converter topologies suitable for high-power dc applications. He has authored more than 20 scientific publications, one tutorial, and filed three patent applications.

In 2020, he has received EPE Outstanding Young Member Award.

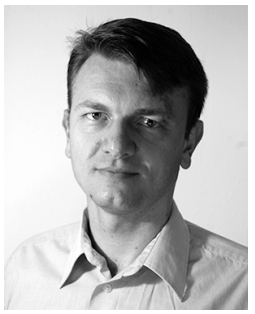

Drazen Dujic (Senior Member, IEEE) is an Assistant Professor and Head of the Power Electronics Laboratory at EPFL. He received the Dipl.Ing. and MSc degrees from the University of Novi Sad, Novi Sad, Serbia in 2002 and 2005, respectively, and the $\mathrm{PhD}$ degree from Liverpool John Moores University, Liverpool, UK in 2008.

From 2003 to 2006, he was a Research Assistant with the Faculty of Technical Sciences at University of Novi Sad. From 2006 to 2009, he was a Research Associate with Liverpool John Moores University. After that he moved to industry and joined ABB Switzerland Ltd, where from 2009 to 2013, he was Scientist and then Principal Scientist with ABB Corporate Research Center in Baden-Dättwil, and from 2013 to 2014 he was R\&D Platform Manager with ABB Medium Voltage Drives in Turgi. He is with EPFL since 2014

His research interests include the areas of design and control of advanced high power electronic systems and high-performance drives, predominantly for the medium voltage applications related to electrical energy generation, conversion and storage. He has authored or co-authored more than 150 scientific publications and has 15 patent applications to his name.

In 2018 he has received EPE Outstanding Service Award and in 2014, the Isao Takahashi Power Electronics Award for Outstanding Achievement in Power Electronics. He is Senior Member of IEEE, EPE Member, and serves as Associate Editor for IEEE Transactions on Power Electronics, IEEE Transactions on Industrial Electronics, IET Electric Power Applications and EPE Journal. 